\section{Medidas directas e indirectas de las funciones ejecutivas en niños con trastorno de espectro autista}

Pérez-Pichardo $\mathrm{MF}^{1}$, Ruz-Sahrur A², Barrera-Morales K ${ }^{1}$, Moo-Estrella J ${ }^{1}$

Resumen

INTRODUCCIÓN: los niños con trastorno del espectro autista (TEA) tienen dificultades en las funciones ejecutivas (FE) pero se han sugerido discrepancias entre las medidas directas e indirectas.

OBJETIVO: conocer si existen diferencias en las funciones ejecutivas mediante medidas directas e indirectas entre niños con y sin TEA.

MÉTODO: se incluyeron 14 varones entre primero y tercero de primaria, 50\% con diagnóstico de TEA y 50\% con desarrollo neurotípico, seleccionados por emparejamiento en edad y grado escolar. El promedio de edad de la muestra fue de 7.43 (DE 0.98) años. Se aplicaron las pruebas de Evaluación neuropsicológica de funciones ejecutivas en niños (ENFEN) y el Inventario de clasificación comportamental de las funciones ejecutivas (BRIEF).

RESULTADOS: se encontraron diferencias significativas en las medidas de las FE indirectas, pero no en las directas, entre los grupos $(p<0.05)$. Así mismo, las correlaciones entre las medidas directas e indirectas fueron mayores en el grupo con TEA.

CONCLUSIONES: los niños con TEA son evaluados con más dificultades en las medidas indirectas de las funciones ejecutivas, pero obtienen puntuaciones similares a los niños con desarrollo neurotípico cuando son evaluados en condiciones estructuradas empleando medidas directas.

PALABRAS CLAVE: trastorno del espectro autista, funciones ejecutivas, desarrollo neurotípico, niños.

Acta Pediatr Mex. 2018 Jan;39(1):13-22.

\section{Direct and indirect measures of executive functions in children with autism spectrum disorder.}

Pérez-Pichardo $\mathrm{MF}^{1}$, Ruz-Sahrur $\mathrm{A}^{2}$, Barrera-Morales $\mathrm{K}^{1}$, Moo-Estrella $\mathrm{J}^{1}$

\section{Abstract}

INTRODUCTION: Children with autistic spectrum disorder (ASD) present difficulties in executive functions (EF), but discrepancies have been suggested between the direct and indirect measures of these functions.
${ }^{1}$ Universidad Autónoma de Yucatán, Laboratorio de Sueño, Facultad de Psicología.

${ }^{2}$ Centro Terapéutico y Educativo Especializado en Desarrollo Infantil (CTDI).

Recibido: 15 de mayo del 2017

Aceptado: 15 de noviembre del 2017

\section{Correspondencia}

Dr. Jesús Moo Estrella

jmestre@correo.uady.mx

Este artículo debe citarse como

Pérez-Pichardo MF, Ruz-Sahrur A, Barrera-Morales K, Moo-Estrella J. Medidas directas e indirectas de las funciones ejecutivas en niños con trastorno de espectro autista. Acta Pediatr Mex. 2018;39(1):13-22. 
OBJECTIVE: To determinate if there are differences in executive functions through direct and indirect measures between children with and without ASD.

METHOD: Included 14 children between first and third grade, $50 \%$ with ASD diagnosis and $50 \%$ with neurotypical development, selectedby pairing age and grade. The average age of the sample was 7.43 (SD 0.98) years-old. The neuropsychologycal evaluation of executive functions in children (ENFEN) and the behavioral rating of executive functions (BRIEF) were applied.

RESULTS: Significant differences between groups were found $(p<0.05)$ in the indirect measures of executive functions, but not in the direct ones. Likewise, correlations were higher between direct and indirect measures in ASD group.

CONCLUSIONS: ASD children were evaluated with more difficulties in indirect measures of behavioral EF, but they obtain similar scores in direct measures compared to children with neurotypical development under controlled conditions.

KEYWORDS: autistic spectrum disorder; executive functions; neurotypical development; children
Correspondence

Dr. Jesús Moo Estrella

jmestre@correo.uady.mx

\section{INTRODUCCIÓN}

El trastorno del espectro autista (TEA) es un trastorno del neurodesarrollo que se caracteriza por déficits persistentes en la comunicación e interacción social en múltiples contextos. Está asociado con patrones de comportamiento, intereses o actividades repetitivas y restringidas. ${ }^{1}$ Aunque algunas personas con TEA pueden vivir de manera independiente y tener una vida productiva, otras necesitan constante atención y apoyo durante toda su vida. ${ }^{2}$ La Organización Mundial de la Salud (OMS) estima que uno de cada 160 niños presenta la condición del espectro autista; ${ }^{2}$ no obstante, las tasas de prevalencia varían entre países. En México la prevalencia de niños con TEA es de $0.87 \% .^{3}$

Las teorías neuropsicológicas para explicar el TEA se han focalizado en las funciones ejecutivas (FE): procesos cognitivos que regulan el comportamiento dirigido hacia metas; dichas funciones permiten el control de los pensamien- tos y acciones en situaciones nuevas o complejas en las cuales no se obtiene un patrón de respuesta adaptativa. Las FE típicamente incluyen las siguientes habilidades cognitivas: inhibición, memoria de trabajo, flexibilidad, planeación y fluidez. ${ }^{4}$ La teoría de la disfunción ejecutiva postula que los individuos con TEA tienen una alteración temprana de la planificación de comportamientos complejos originada por un déficit en la memoria de trabajo. ${ }^{5}$ Alteraciones electrofisiológicas ${ }^{6}$ y anatómicas ${ }^{7}$ en las regiones frontales y su conexión con las regiones temporales y parietales, asociadas con las FE y los síntomas del TEA, respaldan esta teoría. La hipótesis sobre el déficit de las FE en niños con TEA se ha convertido en uno de los tópicos de investigación neuropsicológica más relevantes en el estudio del espectro autista.

En este sentido, diferentes estudios han constatado que las personas con TEA muestran un funcionamiento ejecutivo deficiente. ${ }^{5}$ En general, presentan un desempeño inferior en tareas que 
evalúan atención, planeación, flexibilidad mental y memoria de trabajo. Por otro lado, tienden a desempeñarse mejor en tareas de habilidades gráficas, perceptuales o de reproducción de patrones. ${ }^{8}$

Estas diferencias en el perfil de las funciones ejecutivas (FE) en personas con TEA han sido foco de atención en diversos planteamientos teóricos. Una hipótesis para esclarecer estas diferencias plantea que los resultados sean analizados bajo la clasificación de las funciones ejecutivas "frías" y "calientes". ${ }^{9}$ Esta hipótesis sugiere que las personas con autismo están alteradas primariamente en las FE "calientes" y en menor grado en las FE "frías". ${ }^{10}$ En este sentido, las personas con TEA tienen un desempeño inferior en múltiples componentes de la cognición social (FE "calientes") como la regulación del comportamiento, ${ }^{11}$ la motivación, el reconocimiento de emociones y la inferencia social. ${ }^{11,12}$ En tanto que las "frías" incluyen las dificultades en la planeación, flexibilidad mental, ${ }^{10,13}$ fluidez verbal, ${ }^{8,11,14-16}$ inhibición, memoria de trabajo y solución de problemas..$^{10,13-17}$ No obstante, presentan un mejor desempeño en tareas estructuradas y sistemáticas de habilidades gráficas. ${ }^{9}$

Una segunda hipótesis señala que la principal discrepancia no está entre las FE "calientes" y "frías", sino entre las mediciones directas e indirectas de estas funciones. Las medidas directas incluyen pruebas cuyo único informante o ejecutante es el niño mismo, en tanto que las indirectas incluyen pruebas dirigidas a una variedad de informantes (padres, profesores, compañeros $u$ otras personas que se encuentran en contacto con las personas con TEA).

Los resultados obtenidos con medidas directas han sido empleados con menor frecuencia. Por su parte, los resultados entre la correlación de las medidas directas con las indirectas muestran discrepancias, mientras que las medidas indirectas muestran mayor concordancia con los síntomas clínicos del TEA. Se han encontrado discrepancias entre las medias de ejecución con los reportes realizados por terceros, ${ }^{18}$ por lo cual esta hipótesis aún no cuenta con suficiente respaldo, debido a la diversidad de resultados y a los pocos estudios que permiten analizarla.

\section{OBJETIVO}

Conocer si existen diferencias en las medidas directas e indirectas de las funciones ejecutivas y la correlación entre éstas en niños con y sin TEA.

\section{MATERIALES Y MÉTODOS}

\section{Participantes}

Se incluyeron 14 varones que cursaban entre el primer y tercer grados de primaria con un promedio de edad de 7.43 (DE 0.98) años de edad, el 50\% fueron seleccionados por accesibilidad de un centro terapéutico y educativo especializado en desarrollo infantil, los cuales contaban con un diagnóstico de TEA avalado por un especialista. La otra parte fue seleccionada por emparejamiento en cuanto a la edad y grado escolar, pero con desarrollo neurotípico. Se consideraron como criterios de inclusión: tener el diagnóstico de TEA avalado por un especialista, que estuvieran en el nivel básico de primaria y de sexo indistinto. Como criterio de exclusión se consideró que no tuvieran diagnóstico previo de deficiencia intelectual.

\section{Instrumento}

\section{Medición indirecta de las FE}

El Inventario conductual de funciones ejecutivas (BRIEF, por sus siglas en inglés) fue empleado para la medición indirecta de las FE y aplicado 
a padres. Está compuesto por 86 ítems con tres opciones de respuesta: 1 ) nunca, 2) a veces y 3 ) frecuentemente. Este cuestionario permite tener un panorama del funcionamiento ejecutivo en aspectos cotidianos de los niños dentro del amplio rango de edad de los 5 a los 18 años.

Los 86 ítems del BRIEF se agrupan en ocho dominios de funcionamiento ejecutivo (Cuadro 1). Los resultados se agrupan en dos índices principales: regulación de la conducta y metacognición, además de un índice global compuesto por estas dos dimensiones. También se incluyen dos escalas de validez: de inconsistencia, que indica hasta qué punto el informador contesta coherentemente los ítems parecidos y compara este patrón de respuesta con un grupo normativo y de negatividad, que mide el grado en que el informador responde a ítems seleccionados de modo negativo también en relación a un grupo normativo. ${ }^{19-21}$ El BRIEF, tanto en su versión original como en adaptaciones hispanas, muestra la misma estructura factorial con una consistencia interna de sus factores entre 0.80 y $0.98 .^{19,22}$

\section{Medición directa de las FE}

La Evaluación neuropsicológica de funciones ejecutivas en niños (ENFEN) ${ }^{23}$ se usó para medir directamente las funciones ejecutivas de los niños. Esta prueba se aplica individualmente a niños de entre 6 y 12 años. Permite evaluar el desarrollo madurativo global del niño en edad escolar, poniendo énfasis en su funcionamiento ejecutivo. Incluye seis subpruebas: fluidez fonológica, fluidez semántica, senderos gris, senderos a color, anillas e interferencia (Cuadro 2). Adicionalmente, se usó para la subprueba de anillas una escala de seguimiento de instrucciones utilizando el siguiente formato de respuesta: 0 ) Se siguieron todas las instrucciones; 1) No se siguieron una vez; 2) No se siguieron de dos a cinco veces; 3) No se siguieron de seis a diez veces y 4) No se siguieron de once a 14 veces.

\section{Procedimiento}

Inicialmente se solicitó autorización de los padres para la participación de los niños mediante una carta de participación y consentimiento informado. Las aplicaciones de la ENFEN se realizaron en salones acondicionados, libres de distractores y manteniendo ventilación y luz adecuadas. La duración de la aplicación fue, en promedio, de $48 \pm 2$ minutos en el grupo con TEA y de $30 \pm 3$ minutos para el grupo control. El cuestionario BRIEF fue respondido por los padres de los niños de ambos grupos durante los horarios de clase de los niños. La duración del tiempo de respuesta fue de entre 10 y 15 minutos. No se estableció un tiempo límite para responder las pruebas y ambas fueron aplicadas por dos estudiantes de los últimos semestres de la licenciatura en Psicología, con entrenamiento previo para su aplicación.

El protocolo siguió los estándares éticos de acuerdo con la Norma Oficial Mexicana: NOM004-SSA3-201 en materia de investigación para la salud. A los tutores se les entregó, por escrito, el objetivo del estudio, la justificación, riesgos y beneficios, confidencialidad de sus datos, así como su consentimiento de participación voluntaria y libertad de retirarse en cualquier momento durante el estudio.

Para el análisis de los datos se utilizaron las puntuaciones naturales tanto de la prueba BRIEF como de la ENFEN. Los datos fueron procesados empleando inicialmente estadísticas descriptivas como media (M) y desviación estándar (DE). Para la comparación de los datos entre los grupos se emplearon estadísticas no paramétricas usando la U Mann-Witney. Para establecer la correlación entre las medidas directas y las indirectas en cada grupo se empleó la prueba de correlación Rho de Spearman. El nivel alfa establecido para todas las pruebas estadísticas fue: $\alpha \leq 0.05$. Para el procesamiento de los datos se empleó el programa IBM ${ }^{\circledR}$ SPSS Statistics versión 21. 
Cuadro 1. Descripción, número ítems y puntuación de los dominios del inventario conductual de funciones ejecutivas (BRIEF, por sus siglas en inglés) ${ }^{19}$

\begin{tabular}{|c|c|c|c|}
\hline Dominios BRIEF & Descripción conductual & (Número de ítems) Ejemplos & $\begin{array}{c}\text { Puntuación } \\
\text { mínima-máxima }\end{array}$ \\
\hline Control emocional & $\begin{array}{l}\text { Habilidad para regular sus respuestas emocio- } \\
\text { nales. Un pobre control emocional puede ex- } \\
\text { presarse en forma de explosividad emocional. }\end{array}$ & $\begin{array}{l}\text { (10) "Reacciona de manera } \\
\text { exagerada ante problemas } \\
\text { pequeños", "Se molesta con } \\
\text { mucha facilidad". }\end{array}$ & $10-30$ \\
\hline Memoria de trabajo & $\begin{array}{l}\text { Capacidad para retener información con el } \\
\text { propósito de completar una tarea. Es esencial } \\
\text { para llevar a cabo actividades con muchos } \\
\text { pasos, aritmética o seguir instrucciones com- } \\
\text { plejas. }\end{array}$ & $\begin{array}{l}\text { (10) "Cuando se le dan tres co- } \\
\text { sas para hacer, recuerda solo la } \\
\text { última o la primera", "Cuando } \\
\text { se le manda a buscar algo, se } \\
\text { le olvida qué es lo que fue a } \\
\text { buscar". }\end{array}$ & $10-30$ \\
\hline Iniciación & $\begin{array}{l}\text { Empezar una actividad, independientemente } \\
\text { de generar ideas. }\end{array}$ & $\begin{array}{l}\text { (8) "Necesita que le digan que } \\
\text { inicie una tarea a pesar de que } \\
\text { tiene la intención de hacerla", } \\
\text { "Se queja de no tener nada que } \\
\text { hacer". }\end{array}$ & $8-24$ \\
\hline $\begin{array}{l}\text { Organización de } \\
\text { materiales }\end{array}$ & $\begin{array}{l}\text { Conservar el orden del lugar de trabajo o juego } \\
\text { y de los materiales. }\end{array}$ & $\begin{array}{l}\text { (6) "Deja su cuarto hecho un } \\
\text { desastre", "Deja un rastro de } \\
\text { sus pertenencias a donde quie- } \\
\text { ra que vaya". }\end{array}$ & $6-18$ \\
\hline Flexibilidad & $\begin{array}{l}\text { Capacidad para cambiar fácilmente de situa- } \\
\text { ción, actividad o un aspecto de un problema } \\
\text { a otra si la situación lo demanda, transición, } \\
\text { solución de problemas. }\end{array}$ & $\begin{array}{l}\text { (8) "Se resiste o le cuesta } \\
\text { trabajo aceptar una manera } \\
\text { diferente de resolver problemas } \\
\text { con trabajos escolares, amigos, } \\
\text { mandados", "Se molesta cuan- } \\
\text { do se enfrenta a situaciones } \\
\text { nuevas". }\end{array}$ & $8-24$ \\
\hline $\begin{array}{l}\text { Planeación/Organi- } \\
\text { zación }\end{array}$ & $\begin{array}{l}\text { Anticiparse a eventos futuros, establecerse } \\
\text { metas, desarrollar los pasos adecuados con el } \\
\text { fin de llevar a cabo una actividad de manera } \\
\text { sistemática; entendimiento y comunicación de } \\
\text { ideas principales o conceptos clave. }\end{array}$ & $\begin{array}{l}\text { (12) "Tiene buenas ideas, pero } \\
\text { no las puede plasmar en pa- } \\
\text { pel", "Olvida entregar su tarea, } \\
\text { aun cuando ya está terminada". }\end{array}$ & $12-36$ \\
\hline Monitoreo & $\begin{array}{l}\text { Habilidad para monitorear su desempeño } \\
\text { durante o poco después de terminar una tarea } \\
\text { para asegurar el cumplimiento apropiado de } \\
\text { una meta y para el seguimiento de los efectos } \\
\text { que su conducta afecta a otros. }\end{array}$ & $\begin{array}{l}\text { (8) "Comete errores por no te- } \\
\text { ner cuidado", "No se da cuenta } \\
\text { de que ciertas reacciones } \\
\text { molestan a los demás". }\end{array}$ & $8-24$ \\
\hline Inhibición & $\begin{array}{l}\text { Habilidad para inhibir, resistir o no actuar } \\
\text { impulsivamente. }\end{array}$ & $\begin{array}{l}\text { (10) "Actúa más salvaje o más } \\
\text { infantil que otros niños cuando } \\
\text { se encuentra en grupos (fiestas, } \\
\text { cumpleaños, descansos)", } \\
\text { "Tiene problemas para ponerles } \\
\text { límites a sus acciones". }\end{array}$ & $10-30$ \\
\hline
\end{tabular}

\section{RESULTADOS}

Se presentan los resultados de las medidas indirectas mediante la comparación de los factores del inventario BRIEF entre los grupos.
Se encontraron diferencias, en la mayoría de los factores, excepto en "organización de materiales" $(p>0.5)$ en el cual el grupo con TEA obtuvo una puntuación mayor que el grupo control (Cuadro 3). Por otro lado, en las medidas 
Cuadro 2. Descripción de las subpruebas Evaluación neuropsicológica de funciones ejecutivas en niños (ENFEN) ${ }^{23}$

\begin{tabular}{|c|c|}
\hline & Subpruebas ENFEN \\
\hline Fluidez & $\begin{array}{l}\text { Descripción. Riqueza de lenguaje expresivo, amplitud de vocabulario, memoria verbal, } \\
\text { inteligencia cristalizada, memoria semántica y memoria de trabajo. } \\
\text { Instrucciones. Se asigna un minuto para decir en voz alta tantas palabras como pueda que } \\
\text { empiecen con la letra "M" y otro minuto para las que pertenezcan a la categoría "animales". } \\
\text { Calificación. Número de palabras correctas. }\end{array}$ \\
\hline Senderos & $\begin{array}{l}\text { Descripción. Percepción espacial, eficiencia visomotora, rapidez perceptiva, razonamiento } \\
\text { lógico, capacidad para inhibir, de anticipación y previsión, flexibilidad mental, programa- } \\
\text { ción dual y memoria de trabajo. } \\
\text { Instrucciones. Se muestran dos hojas con números ordenados aleatoriamente. En la pri- } \\
\text { mera, deben unirse del } 20 \text { al } 1 \text { y en la segunda, del } 1 \text { al } 21 \text {, pero alternando los colores } \\
\text { amarillo y rosa. } \\
\text { Calificación. } \\
\frac{\text { Aciertos - (Omisiones }+ \text { Sustituciones) }}{\text { Tiempo (segundos) }} \times 100\end{array}$ \\
\hline Anillas & $\begin{array}{l}\text { Descripción. Capacidad de secuenciación, planificación y previsión de conductas, flexibi- } \\
\text { lidad mental, habilidad para desarrollar y mantener estrategias de solución de problemas } \\
\text { adecuadas al logro de un objetivo, capacidad para descomponer un problema global } \\
\text { en diferentes submetas, para descubrir las reglas de transformación, de estructuración, } \\
\text { orientación espacial y abstracción, memoria prospectiva, operativa y coordinación motora. } \\
\text { Instrucciones. En un tablero de tres ejes verticales deben reproducirse modelos de comple- } \\
\text { jidad creciente presentados en unas láminas colocando una serie de anillas en el mismo } \\
\text { orden que se muestran. } \\
\text { Calificación. Suma total de segundos. }\end{array}$ \\
\hline Interferencia & $\begin{array}{l}\text { Descripción. Atención sostenida y selectiva, capacidad de inhibición y resistencia a la } \\
\text { interferencia. } \\
\text { Instrucciones. El sujeto debe decir en voz alta el color de la tinta en que están impresas } \\
39 \text { palabras divididas equitativamente en } 3 \text { columnas verticales. } \\
\text { Calificación. } \\
\frac{\text { Aciertos - (Omisiones }+ \text { Sustituciones) }}{\text { Tiempo (segundos) }} \times 100\end{array}$ \\
\hline
\end{tabular}

directas (ENFEN) solo se encontraron diferencias en la subprueba de Sendero a color, en la cual el grupo con TEA obtuvo el promedio más bajo (M 5.74, DE 2.51) en comparación con el grupo neurotípico (M 8.66, DE 2.07) (Cuadro 4).

En relación con la escala de seguimiento de instrucciones en anillas se encontraron diferencias $(U 8, p=0.027)$ entre los grupos. El grupo con TEA tuvo mayores dificultades y obtuvo una puntuación más alta en comparación con el grupo control (M 1.85, DE 1.21 vs. M 0.42, DE 0.78). La correlación entre las subpruebas de la batería ENFEN y los factores de la escala BRIEF por grupos se resumen en el Cuadro 5.
El grupo con TEA obtuvo tres correlaciones significativas $(p<0.05)$ : 1$)$ entre sendero gris e inhibición; 2) sendero a color y flexibilidad y 3 ) fluidez semántica y memoria de trabajo. Por su parte, el grupo control obtuvo dos correlaciones significativas: 1 ) entre sendero a color y organización de materiales y 2) entre la prueba de anillas y monitoreo.

\section{DISCUSIÓN}

El estudio planteó conocer si existen diferencias en las funciones ejecutivas mediante medidas directas e indirectas entre niños con y sin TEA. 
Cuadro 3. Comparación de los factores del inventario BRIEF entre grupos

\begin{tabular}{|c|c|c|c|c|c|c|}
\hline & \multicolumn{2}{|c|}{ TEA } & \multicolumn{2}{|c|}{ Control } & \multirow[t]{2}{*}{$\mathbf{U}$} & \multirow[t]{2}{*}{$p$} \\
\hline & $\mathbf{M}$ & DE & $M$ & DE & & \\
\hline $\begin{array}{l}\text { Inhibición } \\
\text { (I) }\end{array}$ & 20.86 & 3.85 & 12.57 & 3.82 & 3.5 & 0.006 \\
\hline $\begin{array}{l}\text { Flexibilidad } \\
\text { (S) }\end{array}$ & 14.86 & 1.77 & 11.14 & 2.48 & 4.5 & 0.010 \\
\hline $\begin{array}{l}\text { Control } \\
\text { emocional } \\
\text { (EC) }\end{array}$ & 20.71 & 4.82 & 14.00 & 3.83 & 6.5 & 0.020 \\
\hline $\begin{array}{l}\text { BRI } \\
(\mathrm{I}+\mathrm{S}+\mathrm{EC})\end{array}$ & 56.43 & 9.81 & 37.71 & 9.39 & 5.5 & 0.014 \\
\hline $\begin{array}{l}\text { Iniciación } \\
\text { (IE) }\end{array}$ & 15.71 & 3.30 & 11.43 & 2.57 & 6 & 0.016 \\
\hline $\begin{array}{l}\text { Memoria } \\
\text { de trabajo } \\
\text { (WM) }\end{array}$ & 21.57 & 3.60 & 14.14 & 4.45 & 5.5 & 0.014 \\
\hline $\begin{array}{l}\text { Planeación } \\
\text { (P) }\end{array}$ & 26.43 & 4.20 & 16.00 & 6.19 & 5 & 0.012 \\
\hline $\begin{array}{l}\text { Organi- } \\
\text { zación de } \\
\text { materiales } \\
(\mathrm{OM})\end{array}$ & 13.86 & 2.61 & 10.86 & 2.91 & 9.5 & 0.052 \\
\hline $\begin{array}{l}\text { Monitoreo } \\
\text { (M) }\end{array}$ & 18.29 & 4.31 & 12.14 & 3.13 & 6 & 0.017 \\
\hline $\begin{array}{l}\text { MI } \\
(\mathrm{IE}+\mathrm{WMP} \\
+\mathrm{OM}+\mathrm{M})\end{array}$ & 95.86 & 14.28 & 64.57 & 17.76 & 5 & 0.012 \\
\hline $\begin{array}{l}\text { GEC } \\
(B R I+M I)\end{array}$ & 152.29 & 20.11 & 102.29 & 26.74 & 5.5 & 0.014 \\
\hline
\end{tabular}

Así mismo se planteó conocer la correlación entre las medidas directas e indirectas en cada grupo. Los resultados indican que, con base en las medidas indirectas, los niños muestran mayores diferencias en las funciones ejecutivas que las que obtienen en sus pruebas directas, como se ha reportado previamente en la literatura. ${ }^{18}$

Las puntuaciones más altas de los niños con TEA, en las medidas indirectas, indican mayor frecuencia de comportamientos disfuncionales percibidos por los padres. Posiblemente la interacción diaria con sus hijos les permite identificar sus conductas problemáticas, que están relacionadas con las funciones ejecutivas (p. ej. memoria de trabajo, control emocional, planeación) en la vida cotidiana. No obstante, los niños con TEA obtienen puntuaciones en las medidas directas similares a los niños con desarrollo neurotípico. Una hipótesis es que los niños con TEA tienen dificultades para regular, mantener y planear su conducta en un entorno social y cambiante como la vida diaria, ${ }^{24}$ pero obtienen mejores resultados en tareas estructuradas y específicas como las que se presentan en las pruebas neuropsicológicas. En este sentido la tendencia a obtener puntuaciones más bajas en sus pruebas directas de funciones ejecutivas puede estar relacionada con las dificultades en el seguimiento de las instrucciones. Durante la aplicación de las pruebas directas los niños del grupo con TEA ejecutaron correctamente la prueba; sin embargo, después de dos o más intentos para realizar la tarea mostraron dificultades para seguir las reglas. Por ejemplo en la prueba de anillas (véase el Cuadro 2) lograron concluir el ejercicio, pero sin seguir las reglas establecidas al principio de la prueba. Por su parte, los niños con desarrollo neurotípico realizaron el ejercicio correctamente y solo fue necesario corregirlos en una ocasión en promedio, posiblemente por una mayor capacidad de beneficiarse de la retroali-

Cuadro 4. Comparación de los factores del inventario BRIEF entre grupos

\begin{tabular}{|c|c|c|c|c|c|c|}
\hline & \multicolumn{2}{|c|}{ TEA } & \multicolumn{2}{|c|}{ Control } & \multirow[t]{2}{*}{$\mathbf{U}$} & \multirow[t]{2}{*}{$p$} \\
\hline & $M$ & DE & $M$ & DE & & \\
\hline $\begin{array}{l}\text { Fluidez } \\
\text { fonoló- } \\
\text { gica }\end{array}$ & 5.28 & 1.97 & 8.00 & 3.91 & 12.50 & 0.121 \\
\hline $\begin{array}{l}\text { Fluidez } \\
\text { semán- } \\
\text { tica }\end{array}$ & 10.85 & 5.84 & 14.71 & 2.13 & 13.50 & 0.159 \\
\hline $\begin{array}{l}\text { Sendero } \\
\text { gris }\end{array}$ & 12.70 & 4.72 & 19.06 & 5.43 & 12.00 & 0.110 \\
\hline $\begin{array}{l}\text { Sendero } \\
\text { a color }\end{array}$ & 5.74 & 2.51 & 8.66 & 2.07 & 8.00 & 0.035 \\
\hline Anillas & 420.57 & 160.67 & 335.00 & 67.53 & 17.00 & 0.338 \\
\hline $\begin{array}{l}\text { Interfe- } \\
\text { rencia }\end{array}$ & 42.02 & 19.18 & 55.19 & 16.06 & 18.00 & 0.406 \\
\hline
\end{tabular}


mentación; no obstante, al corregir a los niños del grupo con TEA y recordarles las reglas ellos seguían perseverando en su comportamiento. Esto refleja lo encontrado en investigaciones anteriores en niños con TEA acerca de las fallas en el mantenimiento de una estrategia y la rigidez cognitiva observada en la repetición de la misma conducta a pesar de ser corregida. ${ }^{8}$

En la medición directa la subprueba senderos a color fue la única que mostró diferencias entre el grupo de niños con TEA y el grupo con desarrollo neurotípico. Las funciones ejecutivas relacionadas con la tarea de sendero a color incluyen flexibilidad, planeación y memoria de trabajo. La diferencia en la ejecución de esta prueba entre los grupos corrobora los resultados de las investigaciones iniciales sobre el perfil neuropsicológico ${ }^{7,8,10,11,13-17}$ y neurofuncional ${ }^{6,7}$ de los niños con TEA.

Es importante destacar que no se puede hablar de una disfunción generalizada de todas las FE, como lo plantea la teoría de Russell, ${ }^{5}$ ya que la mayoría de las subpruebas de medición directa de las FE no mostraron diferencias entre los grupos. Las principales diferencias planteadas en la literatura se relacionan con las dificultades en la regulación emocional y el lenguaje no verbal, las cuales son consideradas como funciones ejecutivas "calientes", incluso cuando se miden mediante medidas directas. ${ }^{9,17,25}$ Por otra parte, las correlaciones entre las medidas directas e indirectas en el grupo con TEA apoyan la hipótesis sobre el papel de las funciones ejecutivas en la regulación del comportamiento en el contexto social, ${ }^{25}$ no obstante, se ha sugerido que las FE están asociadas con funciones comportamentales específicas. ${ }^{26}$

Nuestros datos sugieren que la correlación entre las medidas directas e indirectas, particularmente de las funciones de regulación conductual (inhibición y flexibilidad de la prueba BRIEF) tienen

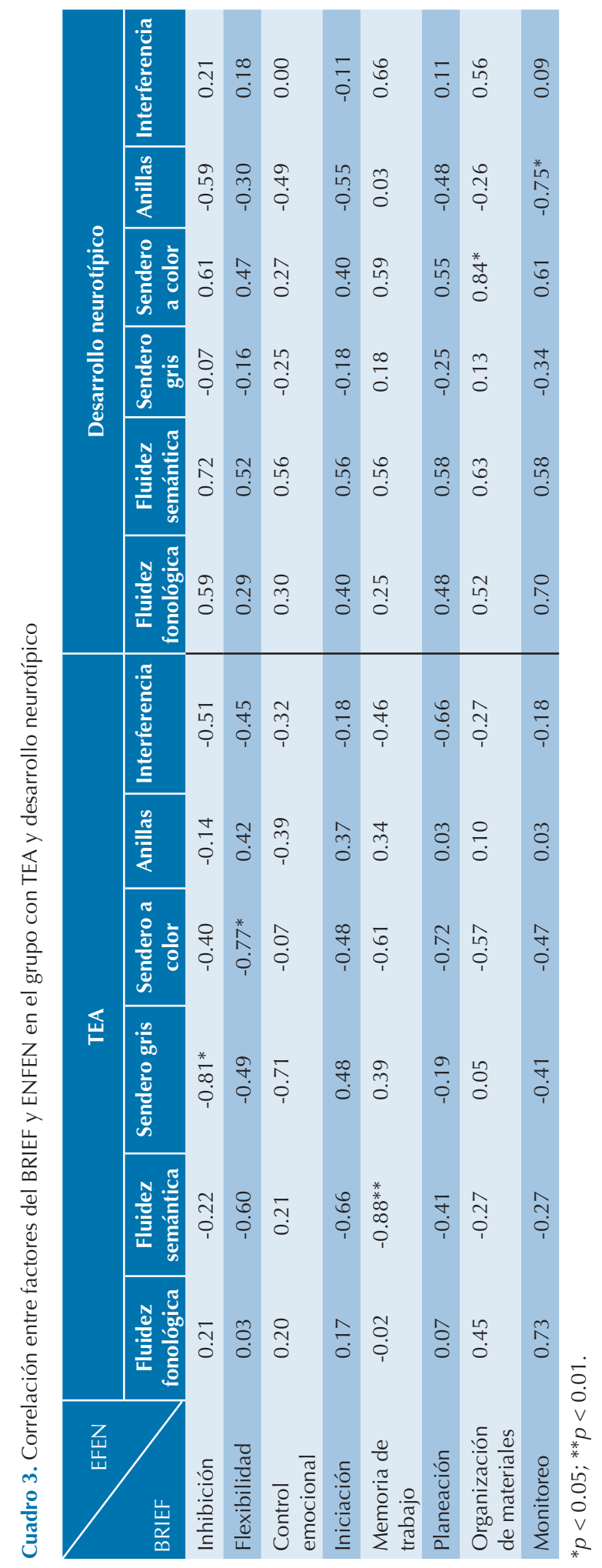


mejor relación con las subpruebas directas que evalúan estos procesos (senderos gris y color) en el grupo con TEA, pero no en el grupo con desarrollo neurotípico. En este sentido, apoyan la hipótesis que plantea la existencia de un vínculo particular entre la regulación conductual social y las funciones ejecutivas en los niños con TEA que no está presente en los niños con desarrollo neurotípico, como se ha propuesto en estudios previos. ${ }^{27}$ Estas correlaciones sirven de base para fundamentar que la intervención sobre las funciones ejecutivas en los niños con TEA puede tener efectos positivos sobre su desarrollo social, con beneficios tanto para el niño como para sus padres. ${ }^{28}$

El estudio incluye diferentes limitaciones. Primera: el tamaño de la muestra que limita los análisis estadísticos para la generalización de los resultados a una población mayor en niños con TEA. Segunda: los grupos no fueron seleccionados del mismo centro educativo, lo cual puede implicar diferencias socioeducativas que no se ven reflejadas en los resultados. Tercera: para los instrumentos utilizados, aun cuando existen datos en versiones en español, no se han realizado investigaciones acerca de sus características psicométricas en la población infantil de la muestra de estudio.

\section{CONCLUSIONES}

1. El reporte comportamental de los padres de niños con TEA muestra diferencias en comparación con el reporte de niños con desarrollo neurotípico.

2. Los niños con TEA son evaluados con más dificultades de inhibición, flexibilidad, iniciación, planeación/organización, monitoreo, memoria de trabajo y control emocional.

3. Las dificultades de organización de materiales pueden considerarse características tanto de los niños neurotípicos como de los niños con TEA.
4. No se encontraron diferencias en la mayoría de las funciones ejecutivas evaluadas directamente entre niños con TEA y neurotípicos.

5. Los niños con TEA tiene dificultades comportamentales en un entorno social y cambiante como la vida diaria, asociadas con las funciones ejecutivas "calientes"; pero obtienen mejores resultados en condiciones controladas y específicas que evalúan las funciones ejecutivas "frías".

6. Las correlaciones sugieren la existencia de un vínculo particular entre las funciones ejecutivas y la regulación comportamental en los niños con TEA, que puede servir de base para la intervención neuropsicológica de niños con TEA.

\section{REFERENCIAS}

1. Asociación Americana de Psiquiatría. Manual Diagnóstico y Estadístico de los Trastornos Mentales: Quinta Edición. 2013 May.

2. Organización Mundial de la Salud. Medidas integrales y coordinadas para gestionar los trastornos del espectro autista. 2013; Obtenido de: http://apps.who.int/iris/bitstream/10665/172324/1/B133_4-sp.pdf

3. Fombonne E, Marcin C, Manero AC, Bruno R, Diaz C, Villalobos $\mathrm{M}$ et al. Prevalence of Autism Spectrum Disorders in Guanajuato, Mexico: The Leon survey. J Autism and Dev Disord. 2016;46(5):1669-1685.

4. Lai CLE, Lau Z, Lui SSY, Lok E, Tam V, Chan Q, Cheng KM, Lam SM, Cheung EFC. Meta-analysis of neuropsychological measures of executive functioning in children and adolescents with high-functioning autism spectrum disorder. Autism Res. 2016 DOI: 10.1002/aur.1723

5. Russell J. El autismo como trastorno de la función ejecutiva. Madrid: Editorial Médica Panamericana. 2000.

6. Han YM, Chan AS. Disordered cortical connectivity underlies the executive function deficits in children with autism spectrum disorders. Res Dev Disabil. 2016;61:19-31.

7. Grecucci A, Rubicondo D, Siugzdaite R, Surian L, Job R. Uncovering the Social Deficits in the Autistic Brain. A Source-Based Morphometric Study. Front Neurosci. 2016 Aug 31;10:388. doi:10.3389/fnins.2016.00388.

8. Talero-Gutiérrez C, Echeverría CM, Sánchez P, Morales G, Vélez-van-Meerbeke A. Trastorno del espectro autista y función ejecutiva. ActaNeurol Colom. 2015;31(3):246-252.

9. Tsermentseli S, Poland S. Cool versus hot executive function: A new approach to executive function. Encephalos. 2016;53:11-14. 
10. Hongwanishkul D, Happaney KR, Lee WSC, Zelazo PD. Assessment of hot and Cool Executive Function in Young Children: Age-Related Changes and Individual Differences. Dev Neuropsychol. 2005;28(2):617-644. DOI: 10.1207/ s15326942dn2802_4.

11. Robinson S, Goddard L, Dritschel B, Wisley M, Howlin P. Executive functions in children with autism spectrum disorders. Brain Cogn. 2009 Dec;71(3):362-8. DOI: 10.1016/j. bandc.2009.06.007.

12. Downs D, Smith T. Emotional understanding, cooperation, and social behavior in high-functioning children with autism. Autism Dev Disord. 2004;34(6). DOI: 10.1007/ s10803-004-5284-0

13. Chan AS, Cheung M, Han YMY, Sze SL, Leung W, Man HS, To CY. Executive function déficits and neural discordance in children with Autism Spectrum Disorder. Clin Neuropsysiol. 2009;120:1107-1115.

14. Etchepareborda MC. Funciones ejecutivas y autismo. Rev Neurol. 2005;41(1):155-162.

15. Ortiz E, Ayala F, Reyes A, López R, Mexicano G. Evaluación de las funciones ejecutivas en niños con trastornos del espectro autista. Rev Neuropsic Lat. 2013;5(4):53-60.

16. Merchán-Naranjo J, Boada L, Rey-Mejías A, Mayoral M, Llorente C, Arango C, Parellada M. La función ejecutiva está alterada en los trastornos del espectro autista, pero no correlaciona con la inteligencia. Rev Psiquiatr Salud Ment. 2015;9(1):39-50. DOI: 10.1016/j.rpsm.2015.10.005.

17. Zimmerman DL, Ownsworth T, O'Donovan A, Roberts J, Gullo MJ. Independence of Hot and Cold Executive Function Deficits in High-Functioning Adults with Autism Spectrum Disorder. Front Hum Neurosci. 2016;10:24. DOI: 10.3389/ fnhum.2016.00024

18. Gómez-Pérez MM, Calero MD, Mata S, Molinero C. Discrepancies between direct and indirect measures of interpersonal and neurocognitive skills in autism spectrum disorder children. J Clin Exp Neuropsychol. 2016;38(8):1-12.

19. Gioia GA, Isquith PK, Guy SC, Kenworthy L. BRIEF: Behavior Rating Inventory of Executive Function: Professional Manual. EE.UU:PAR. 2000
20. Soprano AM. Evaluación de las funciones ejecutivas en el niño. Rev Neurol. 2003;37(1):44-50.

21. Maldonado, MJ. Adaptación del BRIEF (Behavior Rating Inventory of Executive Function) a población española y su utilidad para el diagnóstico del trastorno por déficit de atención-hiperactividad subtipos inatento y combinado. Universidad Complutense de Madrid. 2016.

22. García T, González-Pienda JA, Rodríguez C, Álvarez L. Psychometric characteristics of the BRIEF scale for the assessment of executive functions in Spanish clinical population. Psicothema. 2014;26(1):47-52. DOI: 10.7334/ psicothema2013.149

23. Portellano JA, Martínez R, Zumárraga L. ENFEN: Evaluación Neuropsicológica de las Funciones Ejecutivas en Niños. Madrid: TEA Ediciones. 2009.

24. Nah YH, Poon KK. The perception of social situations by children with autism spectrum disorders. Autism. 2010;15(2):185-203. DOI: 10.1177/1362361309353616.

25. Semrud-Clikeman M, Walkowiak J, Wilkinson A, Minne EP. Direct and indirect measures of social perception, behavior, and emotional functioning in children with Asperger's disorder, nonverbal learning disability, or ADHD. J Abnorm Child Psychol 2010;38(4):509-519.

26. Teunisse JP, Roelofs RL, Verhoeven EW, Cuppen L, Mol J, Berger HJ. Flexibility in children with autism spectrum disorders (ASD): Inconsistency between neuropsychological tests and parent-based rating scales. J Clin Exp Neuropsychol. 2012;34(7):714-723.

27. Leung RC, Vogan VM, Powell TL, Anagnostou E, Taylor MJ. The role of executive functions in social impairment in Autism Spectrum Disorder. Child Neuropsychol. 2016;22(3):336-344.

28. Weiss JA, Viecili MA, Sloman L, Lunsky Y. Direct and indirect psychosocial outcomes for children with autism spectrum disorder and their parents following a parent-involved social skills group intervention. J Can Acad Child Adolesc Psychiatry. 2013;22(4):303-309. 